\title{
On Basis Properties of Function Systems in Lebesgue Spaces
}

\author{
Bilalov B. T. , Garayev T. Z. \\ Institute of Mathematics and Mechanics of NAS of Azerbaijan
}

\begin{abstract}
So me approximative issues related to function systems in Lebesgue spaces are treated in this work, such as the continuation of basis, the non-minimality of basis in subinterval, the relationship between completeness and minimality of sine and cosine type systems. It is proved that the basis properties of sines and cosines type systems in Lebesgue space of functions depend on the number of exponential summands in expressions of these systems.
\end{abstract}

Keywords Completeness, Minimality, Lebesgue Space Classification 2000: 30B60, 42A65, 46B15

\section{Introduction}

The study of approximative properties of function systems in Lebesgue spaces represents special scientific interest for applications in various areas of mathematics. In particular, these matters are important in the spectral theory of differential operators and in the theory of wavelet analysis. Obvious examples are the classical systems of exponentials, sines, cosines, and their perturbation. Approximative properties of these systems in various functional spaces are well studied, and there are extensive bibliographies devoted to them (see, e.g.[1-5]). Relationship between the bas is properties of these systems are known, and it is not difficult to establish it. In general, systems of sines and cosines can be written as follows

$$
\begin{aligned}
& \sin n t=\sum_{k=1}^{r} a_{k}^{s}(t) e^{i \alpha_{k}(t) n}, n \in N ; \\
& \cos n t=\sum_{k=1}^{r} a_{k}^{c}(t) e^{i \alpha_{k}(t) n}, \quad n \in Z_{+},
\end{aligned}
$$

where $r=2, N$ is the set of all positive integers, $Z_{+}=\{0\} \bigcup N$ and

$$
\begin{gathered}
\alpha_{1}(t) \equiv t, \alpha_{2}(t) \equiv-t, \\
a_{1}^{s}(t) \equiv-a_{2}^{s}(t) \equiv \frac{1}{2 i}, \\
a_{1}^{c}(t) \equiv a_{2}^{c}(t) \equiv \frac{1}{2}, \forall t \in[0, \pi] .
\end{gathered}
$$

It is easy to see that the set of values of the functions $\alpha_{1}(t)$ and $\alpha_{2}(t)$ fill up the whole segment $[-\pi, \pi]$,

* Corresponding author:

bilalov.bilal@gmail.com (Bilalov B.T.)

Published online at http://journal.sapub.org/ajms

Copyright (C) 2012 Scientific \& Academic Publishing. All Rights Reserved where the basis properties of the system of exponentials $\left\{e^{\text {int }}\right\}_{n \in Z}(Z$ are integers $)$ are examined. Considering the generalization of this case, we obtain a system of the following form

$$
f_{n}(t) \equiv \sum_{k=1}^{r} a_{k}(t) x_{n}\left(\beta_{k}(t)\right), t \in[a, b], n \in N
$$

It turns out the value of $r=2$ ( i.e. the number of exponential summands) plays an important role in studying of the basis properties of systems of sines and cosines. We will establish some relations between the basis properties of systems $\left\{x_{n}\right\}_{n \in N}$ and $\left\{f_{n}\right\}_{n \in N}$ considered in various Banach spaces. We assume that the system $\left\{x_{n}\right\}_{n \in N}$ is defined on the segment $[c, d]$.

It is interesting that, under natural conditions on functions $a_{k}(t)$ and $\beta_{k}(t), k=\overline{1, r}$, if the system $\left\{x_{n}\right\}_{n \in N}$ forms a basis for $L_{p}(c, d)$, then the system $\left\{f_{n}\right\}_{n \in N}$ is non-minimal in $L_{p}(a, b)$ for $r>1$. This phenomenon does not happen in the case of the system of exponentials $x_{n}(t)=e^{\text {int }}, t \in[-\pi, \pi]$, since in this case we deal with $\left\{e^{\text {int }}\right\}_{n \in N}$ (or $\left\{e^{\text {int }}\right\}_{n \geq 0}$ if a system of cosines is considered), the half of the basis $\left\{e^{i n t}\right\}_{n \in Z}$. Apparently similar problem is considered for the first time. These and other approximative properties of systems are closely related to the matters of continuation of the basis on a wide interval which have previously been considered in[5-7].

\section{Main Assumptions and Auxiliary Facts}


Let $[a, b]$ be a segment on the real axis $R$. As usual, by $L_{p}(a, b), 1 \leq p<+\infty$ we mean a Lebesgue space of functions whose absolute value raised to the $p^{\text {th }}$ power is summable in $(a, b)$. The norm in this space is defined as

$$
\|f\|_{p} \equiv\left(\int_{a}^{b}|f(t)|^{p} d t\right)^{1 / p} .
$$

It is known that $\left(L_{p}(a, b)\right)^{*}$ is isometrically isomorphic to $L_{q}(a, b)$, where $q: \frac{1}{p}+\frac{1}{q}=1$ is a nu mber conjugated to $p$. In other words

$$
\begin{gathered}
\forall l \in L_{p}^{*}(a, b), \quad \exists ! f \in L_{q}(a, b): \\
I(x)=\int_{a}^{b} x(t) \overline{f(t)} d t, \forall x \in L_{p}(a, b) .
\end{gathered}
$$

Let us state some ideas from the theory of bases. Let $X$ be some Banach space and $X^{*}$ be its conjugate. We denote by $L[M]$ the linear span of the set $M \subset X$, and $\bar{M}$ will be the closure of $M$ in $X$. We will assume that all the considered spaces are complex.

Definition 1. System $\left\{x_{n}\right\}_{n \in N} \subset X$ is called complete in $X$ if $\overline{L\left[\left\{x_{n}\right\}_{n \in N}\right]}=X$.

Definition 2. System $\left\{x_{n}\right\}_{n \in N} \subset X$ is called minimal in $X$ if $x_{k} \notin \overline{L\left[\left\{x_{n}\right\}_{n \neq k}\right]}, \quad \forall k \in N$.

The following criteria of these properties are well known

Statement 1. System $\left\{x_{n}\right\}_{n \in N} \subset X$ is complete in $X$ if and only iffrom

$$
x^{*} \in X^{*}: x^{*}\left(x_{n}\right)=0, \forall n \in N \text {, it follows that }
$$
$x^{*}=0$.

Statement 2. System $\left\{x_{n}\right\}_{n \in N} \subset X$ is minimal in $X$ if and only if $\exists\left\{x_{n}^{*}\right\}_{n \in N} \subset X^{*}: x_{n}^{*}\left(x_{k}\right)=\delta_{n k}$, $\forall n, k \in N$, where $\delta_{n k}$ is the Kronecker symbol.

Recall also the definition of the basis

Definition 3. System $\left\{x_{n}\right\}_{n \in N} \subset X$ forms a basis for $X$ iffor $\forall x \in X \quad \exists !\left\{\lambda_{n}\right\}_{n \in N} \subset \mathbb{C}$ :

$$
x=\sum_{n=1}^{\infty} \lambda_{n} x_{n} \text {, where } \mathbb{C} \text { is a field of complex }
$$
numbers.

More details of these and other facts from the theory of bases can be found in the monographs [8-11]. Thus, the completeness of the system $\left\{x_{n}\right\}_{n \in N} \subset L_{p}(a, b)$ is equivalent to the fact that $\int_{a}^{b} x(t) \overline{f(t)} d t=0, \quad \forall n \in N$, $f \in L_{q}(a, b)$, implies $f=0$. Minimality of the system $\left\{x_{n}\right\}_{n \in N} \subset L_{p}(a, b)$ means that $\exists\left\{y_{n}\right\}_{n \in N} \subset L_{q}(a, b)$ :

$$
\int_{a}^{b} x_{n}(t) \overline{y_{m}(t)} d t=\delta_{n m}, \quad \forall n, m \in N .
$$

We make the following basic assumptions concerning functions $a_{k}(t)$ and $\left.\beta_{k}(t), \quad k=\overline{1, r}: \quad \alpha \quad\right)$ $\beta_{k}(t), k=\overline{1, r}$, are piecewise smooth, monotonous functions on $(a, b)$; moreover, $\beta_{k}\{(a, b)\} \subset[c, d]$, $k=\overline{1, r}$, and $\beta_{k}\{(a, b)\} \cap \beta_{i}\{(a, b)\}=\{\varnothing\}$ for $k \neq i$, where $f\{I\}$ denotes the image of the set $I$, i.e. $f\{I\} \equiv\{y: y=f(t), \forall t \in I\}$.

$\beta$ ) $\quad \beta_{k}^{\prime}(t)$ and $a_{k}(t), k=\overline{1, r}$ are measurable functions on $(a, b)$ and the inequality $\sup _{(a, b)} \operatorname{vrai}\left\{\left|a_{k}(t)\right|^{ \pm 1} ;\left|\beta_{k}^{\prime}(t)\right|^{ \pm 1}\right\}<+\infty, \quad k=\overline{1, r}$, holds, where $[f(t)]^{\prime}$ denotes the derivative of $f$ in $t$.

Throughout this paper we will use the notation $M^{c}[\mathscr{D}] \equiv \mathscr{D} \backslash M$.

\subsection{Continuation of the Basis}

Let systems $\left\{x_{n}\right\}_{n \in N}$ and $\left\{y_{n}\right\}_{n \in N}$ form bases for spaces $L_{p}(a, b)$ and $L_{p}(b, c), a<b<c$, respectively. By $\left\{f_{n}\right\}_{n \in N}$ and $\left\{g_{n}\right\}_{n \in N}$ we denote the corresponding biorthogonal systems. Let's consider arbitrary functions $\lambda(t)$ and $\mu(t)$ :

$$
|\lambda(t)|^{ \pm 1} \in L_{\infty}(a, b) ;|\mu(t)|^{ \pm 1} \in L_{\infty}(b, c) .
$$

We introduce the following functions

$$
\begin{gathered}
z_{n}(t) \equiv \begin{cases}\alpha_{1} \lambda(t) x_{n}(t), & t \in[a, b], \\
\beta_{1} \mu(t) y_{n}(t), & t \in[b, c],\end{cases} \\
w_{n}(t) \equiv\left\{\begin{array}{l}
\alpha_{2} \lambda(t) x_{n}(t), t \in[a, b], \\
\beta_{2} \mu(t) y_{n}(t), t \in[b, c], \quad \forall n \in N,
\end{array}\right.
\end{gathered}
$$

where

$$
\Delta=\operatorname{det}\left|\begin{array}{ll}
\alpha_{1} & \beta_{1} \\
\alpha_{2} & \beta_{2}
\end{array}\right| \neq 0 \text {. }
$$

Consider the double system

$$
\left\{z_{n}(t) ; w_{n}(t)\right\}_{n \in N}
$$

This system is minimal in $L_{p}(a, c)$, and the system $\left\{\varphi_{n} ; \psi_{n}\right\}_{n \in N}$ biorthogonal to it has the following form

$$
\varphi_{n}(t) \equiv\left\{\begin{array}{l}
\beta_{2} \lambda^{-1}(t) f_{n}(t), t \in[a, b], \\
-\alpha_{2} \mu^{-1}(t) g_{n}(t), t \in[b, c],
\end{array}\right.
$$




$$
\psi_{n}(t) \equiv\left\{\begin{array}{l}
\beta_{1} \lambda^{-1}(t) f_{n}(t), t \in[a, b] \\
-\alpha_{1} \mu^{-1}(t) g_{n}(t), t \in[b, c] .
\end{array}\right.
$$

In fact

$$
\begin{gathered}
\int_{a}^{c} z_{n}(t) \overline{\varphi_{m}(t)} d t=\frac{1}{\Delta} \alpha_{1} \beta_{2} \delta_{n m}-\frac{1}{\Delta} \beta_{1} \alpha_{2} \delta_{n m}= \\
=\frac{1}{\Delta}\left(\alpha_{1} \beta_{2}-\beta_{1} \alpha_{2}\right) \delta_{n m}=\delta_{n m}, \\
\int_{a}^{c} z_{n}(t) \overline{\psi_{m}(t)} d t=\frac{1}{\Delta} \alpha_{1} \beta_{1} \delta_{n m}-\frac{1}{\Delta} \beta_{1} \alpha_{1} \delta_{n m}=0 .
\end{gathered}
$$

Similarly we can show that

$$
\begin{gathered}
\int_{a}^{c} w_{n}(t) \overline{\varphi_{m}(t)} d t \\
=0, \int_{a}^{c} w_{n}(t) \overline{\psi_{m}(t)} d t=\delta_{n m} \\
\forall n, m \in N .
\end{gathered}
$$

Now let us prove the completeness of the system (2) in $L_{p}(a, c)$. Let the following relations be true for some $f(t) \in L_{q}(a, c)$ :

$$
\int_{a}^{c} z_{n}(t) \overline{f(t)} d t=0, \int_{a}^{c} w_{n}(t) \overline{f(t)} d t=0, \quad \forall n \in N .
$$

We have

$$
\left.\begin{array}{l}
\alpha_{1} \int_{a}^{b} \lambda(t) x_{n}(t) \overline{f(t)} d t+\beta_{1} \int_{a}^{b} \mu(t) y_{n}(t) \overline{f(t)} d t=0, \\
\alpha_{2} \int_{a}^{b} \lambda(t) x_{n}(t) \overline{f(t)} d t+\beta_{2} \int_{a}^{b} \mu(t) y_{n}(t) \overline{f(t)} d t=0, \forall n \in N .
\end{array}\right\}
$$

From $\Delta \neq 0$ it follows that

$$
\int_{a}^{b} \lambda(t) x_{n}(t) \overline{f(t)} d t=0 \quad, \quad \int_{b}^{c} \mu(t) y_{n}(t) \overline{f(t)} d t=0,
$$

$\forall n \in N$.

Consequently, $f(t)=0$ on $(a, c)$, and this proves the completeness of the system(2) in $L_{p}(a, c)$.

Let us consider $\forall f \in L_{p}(a, c)$ and the partial sum

$$
S_{n, m}(t)=\sum_{k=1}^{n} a_{k}^{(1)} z_{k}(t)+\sum_{k=1}^{m} a_{k}^{(2)} w_{k}(t)
$$

where

$$
\begin{gathered}
a_{k}^{(1)}=\int_{a}^{c} f(t) \overline{\varphi_{k}(t)} d t, \\
a_{k}^{(2)}=\int_{a}^{c} f(t) \overline{\psi_{k}(t)} d t, \quad \forall k \in N
\end{gathered}
$$

Denote

$$
\begin{gathered}
c_{k}=\int_{a}^{b} f(t) \lambda^{-1}(t) \overline{f_{k}(t)} d t, \\
d_{k}=\int_{b}^{c} f(t) \mu^{-1}(t) \overline{g_{k}(t)} d t, \quad \forall k \in N
\end{gathered}
$$

Then

$$
\begin{gathered}
a_{k}^{(1)}=\frac{1}{\Delta}\left(\beta_{2} c_{k}-\alpha_{2} d_{k}\right), a_{k}^{(2)}=-\frac{1}{\Delta}\left(\beta_{1} c_{k}-\alpha_{1} d_{k}\right), \\
\forall k \in N .
\end{gathered}
$$

Let $t \in[a, b]$. In this case we have

$$
\begin{gathered}
S_{m, m}(t)=\frac{1}{\Delta} \sum_{k=1}^{m}\left(\beta_{2} c_{k}-\alpha_{2} d_{k}\right) \alpha_{1} \lambda(t) x_{k}(t)- \\
-\frac{1}{\Delta} \sum_{k=1}^{m}\left(\beta_{1} c_{k}-\alpha_{2} d_{k}\right) \alpha_{2} \lambda(t) x_{k}(t)= \\
=\frac{1}{\Delta} \sum_{k=1}^{m}\left(\alpha_{1} \beta_{2}-\beta_{1} \alpha_{2}\right) c_{k} \lambda(t) x_{k}(t)= \\
=\sum_{k=1}^{m} c_{k} \lambda(t) x_{k}(t) .
\end{gathered}
$$

Similarly, for $t \in[b, c]$ we have

$$
\begin{gathered}
S_{m, m}(t)=\frac{1}{\Delta} \sum_{k=1}^{m}\left(\beta_{2} c_{k}-\alpha_{2} d_{k}\right) \beta_{1} \mu(t) y_{k}(t)- \\
-\frac{1}{\Delta} \sum_{k=1}^{m}\left(\beta_{1} c_{k}-\alpha_{1} d_{k}\right) \beta_{2} \mu(t) y_{k}(t)= \\
=\frac{1}{\Delta} \sum_{k=1}^{m}\left(-\beta_{1} \alpha_{2}+\alpha_{1} \beta_{2}\right) d_{k} \mu(t) y_{k}(t)= \\
\sum_{k=1}^{m} d_{k} \mu(t) y_{k}(t) .
\end{gathered}
$$

From these relations it follows that

$$
\int_{a}^{c}\left|S_{m, m}(t)-f(t)\right|^{p} d t \rightarrow 0 \text { as } \quad m \rightarrow \infty,
$$

and thus, the double system (2) forms a basis for $L_{p}(a, c)$ if summation is made symmetrically, i.e. this system forms a symmetrical basis for $L_{p}(a, c)$.

\subsection{Some Approxi mative Properties of Function Systems in Lebesgue Spaces}

By mes $G$ we mean the Lebesgue measure of the set $G \subset R$. All the subsets of real axis we consider are assumed to be Lebesgue measurable. It is easily seen that if the system $\left\{x_{n}\right\}_{n \in N}$ is minimal in $L_{p}(I)$, then it is also minimal in $L_{p}(J): J \supset I$; and if it is complete in $L_{p}(I)$, then it is 
also complete in $L_{p}(J),: J \subset I$. An interesting fact should be noted that the system $\left\{x_{n}\right\}_{n \in N}$ can be complete and minimal at the same time in $L_{p}(J)$ and in $L_{p}(I)$ for mes $(J \backslash I)>0, I \subset J$. Relevant nontrivial example can be found e.g. in [6]. In the case of basis we have the following

Lemma 1. If the system $\left\{x_{n}\right\}_{n \in N}$ forms a basis for $L_{p}(I), \quad p \geq 1$, then it is nonminimal in $L_{p}(J)$ for $J \subset I: \operatorname{mes}(I \backslash J)>0$.

In fact, let this system be minimal in $L_{p}(J)$, and let $\left\{y_{n}\right\}_{n \in N} \subset L_{q}(J)$ be a corresponding biorthogonal system. Assume

$$
z_{n}(t) \equiv\left\{\begin{array}{l}
y_{n}(t), t \in J, \\
0, t \in I \backslash J, n \in N .
\end{array}\right.
$$

Evidently, $\left\{z_{n}\right\}_{n \in N}$ is a system biorthogonal to $\left\{x_{n}\right\}_{n \in N}$ in $L_{p}(I)$. Taking the function

$$
f(t) \equiv\left\{\begin{array}{l}
0, t \in J, \\
1, t \quad \in I \backslash J,
\end{array}\right.
$$

(without loss of generality, it can be assumed that mes $(I \backslash J)<+\infty)$, we obtain that its biorthogonal series doesn't converge to it in $L_{p}(I)$, and this is contrary to lemma's assumption that the system $\left\{x_{n}\right\}_{n \in N}$ forms a basis for $L_{p}(I)$.

Moreover, a basis in $L_{p}(I)$ can be complete also in $L_{p}(J)$ for $I \subset J: \operatorname{mes}(J \backslash I)>0$ (it is minimal in $L_{p}(J)$, of course). Let us give an appropriate example. Let us take an arbitrary orthonormal basis $\left\{x_{n}\right\}_{n \in N}$ in $L_{2}(I)$. Let $\left\{y_{n}\right\}_{n \in N}$ be some complete system in $L_{2}(J \backslash I)$ (in case when $I, J$ are intervals, we can easily make such a choice). Assume $y_{n}^{k}=y_{n}, \forall k, n \in N$, and construct a new system $\left\{z_{n}\right\}_{n \in N}$ in the following way

$$
\begin{gathered}
z_{1}=y_{1}^{1} ; \quad z_{2}=y_{1}^{2} ; \quad z_{3}=y_{2}^{1} ; \\
z_{4}=y_{3}^{1} ; z_{5}=y_{2}^{2} ; \quad z_{6}=y_{1}^{3} ; \ldots
\end{gathered}
$$

Let us consider the system $\left\{w_{n}\right\}_{n \in N}$ defined by the expression

$$
w_{n}(t)=\left\{\begin{array}{l}
x_{n}(t), t \in I, \\
z_{n}(t), t \in J \backslash I, n \in N .
\end{array}\right.
$$

It is obvious that $\left\{w_{n}\right\}_{n \in N}$ forms a basis for $L_{2}(I)$. Show that it is complete in $L_{2}(J)$. Let

$$
\int_{J} w_{n}(t) \overline{f(t)} d t=0, \quad \forall n \in N,
$$

for some $f \in L_{2}(J)$. It follows from construction of $\left\{z_{n}\right\}_{n \in N}$ and (3) that for $\forall n \in N, \exists\left\{n_{m}\right\}_{m \in N} \subset N$ :

$$
\int_{I} x_{n_{m}}(t) \overline{f(t)} d t=-\int_{J \backslash I} y_{n}(t) \overline{f(t)} d t, \quad \forall m \in N .
$$

Evidently, $\lim _{m \rightarrow \infty} \int_{I} x_{n_{m}}(t) \overline{f(t)} d t=0$, and, as a result, $\int_{J \backslash I} y_{n}(t) \overline{f(t)} d t=0, \quad \forall n \in N$. It follows from completeness of the system $\left\{y_{n}\right\}_{n \in N}$ in $L_{2}(J \backslash I)$ that $f(t)=0$ on $J \backslash I$. The further reasoning is obvious.

\section{Main Results}

\subsection{Single Case}

We proceed to the main results. Let us consider the system (1). The following theorem is true.

Theorem 1. Let the conditions $\alpha$ ), $\beta$ ) be fulfilled. Then : 1) it follows from the completeness of the system $\left\{x_{n}\right\}_{n \in N}$ in $L_{p}(c, d)$ that the system $\left\{f_{n}\right\}_{n \in N}$ is complete in $L_{p}(a, b)$; 2) from minimality of the system $\left\{f_{n}\right\}_{n \in N}$ in $L_{p}(a, b)$ it follows that the system $\left\{x_{n}\right\}_{n \in N}$ is minimal in $L_{p}(c, d), p \geq 1$.

Proof. Let us take any function $g \in L_{q}(a, b)$ and consider

$$
I_{n}=\int_{a}^{b} f_{n}(t) \bar{g}(t) d t, \quad n \in N .
$$

Without loss of generality, we will assume that all functions $\beta_{k}$ are increasing. We denote by $\beta_{k}^{-1}(\tau)$ an inverse of the function $\tau=\beta_{k}(t)$. Let $\beta_{k}^{-}=\beta_{k}(a)$, $\beta_{k}^{+}=\beta_{k}(b) \quad$. We set $G(\tau) \equiv g\left(\beta_{k}^{-1}(\tau)\right)$, $\forall \tau \in\left(\beta_{k}^{-}, \beta_{k}^{+}\right), k=\overline{1, r}$. Let us assume $G(\tau) \equiv 0$, $\forall \tau \in I_{\beta}^{c}[(c, d)]$, where $I_{\beta} \equiv \bigcup_{k=1}^{r}\left(\beta_{k}^{-}, \beta_{k}^{+}\right)$, and introduce the function $A(\tau)$ :

$$
A(\tau) \equiv \begin{cases}a_{k}\left(\beta_{k}^{-1}(\tau)\right)\left[\beta_{k}^{-1}(\tau)\right]^{\prime}, & \tau \in\left(\beta_{k}^{-}, \beta_{k}^{+}\right), k=\overline{1, r}, \\ 0 & , \tau \in I_{\beta}^{c}[(c, d)] .\end{cases}
$$

Under these notations, the integrals $I_{n}$ can be written as follows

$$
I_{n}=\int_{c}^{d} A(\tau) \bar{G}(\tau) x_{n}(\tau) d \tau
$$

The validity of the theorem follows directly from (4) and 
(5). In case of minimality, the systems $\left\{x_{n}^{*}\right\}_{n \in N}$ and $\left\{f_{n}^{*}\right\}_{n \in N}$, are biorthogonal to the systems $\left\{x_{n}\right\}_{n \in N}$ and $\left\{f_{n}\right\}_{n \in N}$, respectively, are related by the following formula $x_{n}^{*}(\tau) \equiv \begin{cases}f_{n}^{*}\left(\beta_{k}^{-1}(\tau)\right) \bar{a}_{k}\left(\beta_{k}^{-1}(\tau)\right)\left[\beta_{k}^{-1}(\tau)\right]^{\prime}, \tau \in\left(\beta_{k}^{-}, \beta_{k}^{+}\right), k=\overline{1, r},(6) \\ 0 & , \tau \in I_{\beta}^{c}[(c, d)] .\end{cases}$

The theorem is proved.

Theorem 2. Let the conditions $\alpha$ ), $\beta$ ) be fulfilled. Then, if the system $\left\{x_{n}\right\}_{n \in N}$ forms a basis for $L_{p}(c, d), p \geq 1$, and $I_{\beta}^{c}[(c, d)]$ contains a nontrivial interval ( i.e. mes $\left.I_{\beta}^{c}[(c, d)]>0\right)$, then the system $\left\{f_{n}\right\}_{n \in N}$ is nonminimal in $L_{p}(a, b)$.

The validity of the theorem follows directly from Lemma 1 and (6). The following result was absolutely unexpected for the authors.

Theorem 3. Let the conditions $\alpha$ ), $\beta$ ) be fulfilled and $r>1$. Then if $\left\{x_{n}\right\}_{n \in N}$ forms a basis for $L_{p}(c, d)$, then the system $\left\{f_{n}\right\}_{n \in N}$ is nonminimal in $L_{p}(a, b)$, $1<p<+\infty$.

Proof. Let $\left\{x_{n}^{*}\right\}_{n \in N}$ be a systembiorthogonalto $\left\{x_{n}\right\}_{n \in N}$. It is evident that the system $\left\{x_{n}^{*}\right\}_{n \in N}$ forms a basis for $L_{q}(c, d)$. Consequently, this system is nonminimal in $L_{q}(J)$, where $J \subset(c, d), J \neq(c, d)$ is any interval. Assume that the system $\left\{f_{n}\right\}_{n \in N}$ is minimal in $L_{p}(a, b)$ and $\left\{f_{n}^{*}\right\}_{n \in N}$ is a system biorthogonal to it. The uniqueness of a system biotrhogonal to the complete one and the relation (6) imply that

where

$$
x_{n}^{*}(\tau) \equiv \begin{cases}b_{k}\left(\beta_{k}^{-1}(\tau)\right) f_{n}^{*}\left(\beta_{k}^{-1}(\tau)\right), \tau \in\left(\beta_{k}^{-}, \beta_{k}^{+}\right), k=\overline{1, r} \\ 0 \quad, \tau \in I_{\beta}^{c}[(c, d)]\end{cases}
$$

$$
b_{k}\left(\beta_{k}^{-1}(\tau)\right) \equiv \begin{cases}\bar{a}_{k}\left(\beta_{k}^{-1}(\tau)\right)\left[\left(\bar{\beta}_{k}^{-1}(\tau)\right)\right]^{\prime}, & \tau \in\left(\beta_{k}^{-}, \beta_{k}^{+}\right), k=\overline{1, r}, \\ 0 \quad, \quad \tau \in I_{\beta}^{c}[(c, d)] .\end{cases}
$$

Without loss of generality, we will as sume that $I_{\beta} \equiv(c, d)$. Since doing otherwise we would have, by virtue of Theorem 2 , that the system $\left\{f_{n}\right\}_{n \in N}$ is nonminimal in $L_{p}(a, b)$. Let us introduce the function

We have

$$
v_{k}(\tau) \equiv \beta_{k}\left(\beta_{1}^{-1}(\tau)\right), d_{k}(\tau) \equiv b_{k}\left(\beta_{1}^{-1}(\tau)\right) \bar{\beta}_{k}^{\prime}\left(\beta_{1}^{-1}(\tau)\right)\left[\left(\bar{\beta}_{k}^{-1}(\tau)\right)\right]^{\prime}, \tau \in\left(\beta_{1}(a), \beta_{1}(b)\right)
$$

$$
\int_{\beta_{k}(a)}^{\beta_{k}(b)} x_{n}(\tau) \bar{x}_{m}^{*}(\tau) d \tau=\int_{\beta_{k}(a)}^{\beta_{k}(b)} x_{n}(\tau) \bar{b}_{k}\left(\beta_{k}^{-1}(\tau)\right) \bar{f}_{m}^{*}\left(\beta_{k}^{-1}(\tau)\right) d \tau=\int_{\beta_{1}(a)}^{\beta_{1}(b)} x_{n}\left(v_{k}(\tau)\right) \bar{d}_{k}(\tau) \bar{f}_{m}^{*}\left(\beta_{1}^{-1}(\tau)\right) d \tau .
$$

On the other hand

As a result we obtain

$$
f_{m}^{*}\left(\beta_{1}^{-1}(\tau)\right) \equiv \frac{x_{m}^{*}(\tau)}{b_{1}\left(\beta_{1}^{-1}(\tau)\right)}, \quad \tau \in\left(\beta_{1}(a), \beta_{1}(b)\right)
$$

$$
\int_{\beta_{k}(a)}^{\beta_{k}(b)} x_{n}(\tau) \bar{x}_{m}^{*}(\tau) d \tau \equiv \int_{\beta_{1}(a)}^{\beta_{1}(b)} \frac{\bar{d}_{k}(\tau)}{\overline{b_{1}}\left(\beta_{1}^{-1}(\tau)\right)} x_{n}\left(v_{k}(\tau)\right) \bar{x}_{m}^{*}(\tau) d \tau
$$

Summing this equality over $k$ from 2 to $r$, we have

$$
\sum_{k=2}^{r} \int_{\beta_{k}(a)}^{\beta_{k}(b)} x_{n}(\tau) \bar{x}_{m}^{*}(\tau) d \tau=\int_{\beta_{1}(a)}^{\beta_{1}(b)} \sum_{k=2}^{r} \eta_{k}(\tau) x_{n}\left(v_{k}(\tau)\right) \bar{x}_{m}^{*}(\tau) d \tau,
$$

where

It is obvious that

$$
\eta_{k}(\tau) \equiv \frac{\bar{d}_{k}(\tau)}{\bar{b}_{1}\left(\beta_{1}^{-1}(\tau)\right)}, \quad \tau \in\left(\beta_{1}(a), \beta_{1}(b)\right)
$$




$$
\sum_{k=2}^{r} \int_{\beta_{k}(a)}^{\beta_{k}(b)} x_{n}(\tau) \bar{x}_{m}^{*}(\tau) d \tau+\int_{\beta_{1}(a)}^{\beta_{1}(b)} x_{n}(\tau) \bar{x}_{m}^{*}(\tau) d \tau=\delta_{n m} .
$$

Taking into account this expression in (7), we obtain

where

$$
\int_{\beta_{1}(a)}^{\beta_{1}(b)} z_{n}(\tau) \bar{x}_{m}^{*}(\tau) d \tau=\delta_{n m}
$$

$$
z_{n}(\tau) \equiv x_{n}(\tau)-\sum_{k=2}^{r} \eta_{k}(\tau) x_{n}\left(v_{k}(\tau)\right), \quad \tau \in\left(\beta_{1}(a), \beta_{1}(b)\right)
$$

It is easy to see that $\left\{z_{n}\right\}_{n \in N} \subset L_{p}\left(\beta_{1}(a), \beta_{1}(b)\right)$, and, consequently, $\left\{x_{n}^{*}\right\}_{n \in N}$ is minimal in $L_{q}\left(\beta_{1}(a), \beta_{1}(b)\right)$. Evidently, $\left[\beta_{1}(a), \beta_{1}(b)\right] \subset(c, d)$ for $r>1$. So we have a contradiction by virtue of Lemma 1 .

The theorem is proved.

Corollary 1. System $\sin n t, \quad n \in N, n \neq 4 k+2, \forall k \geq 1$, is complete $i \quad L_{p}\left(\frac{\pi}{4}, \frac{3 \pi}{4}\right), \quad p \geq 1$.

In fact, the apparent equality

$$
\sin n t+\sin n\left(t+\frac{\pi}{2}\right)=2 \cos \frac{\pi n}{4} \sin n\left(t+\frac{\pi}{4}\right)
$$

and Theorem 1 imply that the system $\left\{\sin n\left(t+\frac{\pi}{4}\right)\right\}_{n \in N, n \neq 4 k+2}$ is complete in $L_{p}\left(0, \frac{\pi}{2}\right)$.

\subsection{Double Case}

The similar conclusions can be made for the following function systems

$$
f_{n}^{ \pm}(t) \equiv \sum_{k=1}^{r}\left[b_{k}^{(1)}(t) x_{n}^{+}\left(\omega_{k}(t)\right) \pm b_{k}^{(2)}(t) x_{n}^{-}\left(-\omega_{k}(t)\right)\right]
$$

In this case we assume that the functions $x_{n}^{ \pm}(t), \forall n \in N$, are defined on the segment $[-c, c]$. Let the following conditions be satisfied:

r) $\omega_{k}(t), k=\overline{1, r}$, are piecewise smooth, monotonous functions on $[a, b]$ with $\omega_{k}\{(a, b)\} \subset[0, c], k=\overline{1, r}$; mes $E_{k i}=0$ for $k \neq i$, where $\left.E_{k i}=\omega_{k}\{(a, b)\} \cap \omega_{i}\{(a, b)\} ; \eta\right) \omega_{k}^{\prime}(t), b_{k}^{(1)}(t), b_{k}^{(2)}(t), k=\overline{1, r}$, are measurable functions on $(a, b)$ with

$$
\sup \operatorname{vrai}_{(a, b)}\left\{\left|b_{k}^{(1)}(t)\right|^{ \pm 1} ;\left|b_{k}^{(2)}(t)\right|^{ \pm 1} ;\left|\omega_{k}^{\prime}(t)\right|^{ \pm 1}\right\}<+\infty, k=\overline{1, r}
$$

Assume $I_{\omega}^{ \pm}= \pm \bigcup_{k=1}^{r} \omega_{k}\{(a, b)\}$. Introduce $F_{n}(\tau) \equiv\left\{\begin{array}{l}{\left[\omega_{k}^{-1}(\tau)\right]^{\prime} b_{k}^{(1)}\left(\omega_{k}^{-1}(\tau)\right) x_{n}^{+}(\tau), \tau \in\left(\omega_{k}(a), \omega_{k}(b)\right), k=\overline{1, r},} \\ {\left[\omega_{k}^{-1}(-\tau)\right]^{\prime} b_{k}^{(2)}\left(\omega_{k}^{-1}(-\tau)\right) x_{n}^{-}(\tau), \tau \in\left(-\omega_{k}(b),-\omega_{k}(a)\right), k=\overline{1, r}, \text { and consider the follo wing double systems }}\end{array}\right.$

$$
\begin{gathered}
\left\{F_{n}(\tau) ; F_{n}(-\tau)\right\}_{n \in N}, \\
1 \cup\left\{F_{n}(\tau) ; F_{n}(-\tau)\right\}_{n \in N} .
\end{gathered}
$$

It is easy to prove the following

Theorem 4. Let the conditions $\gamma), \eta)$ be fulfilled. Then: 1) if the system $\left(8_{1}\right),\left(\left(8_{2}\right)\right)$ is complete in $L_{p}(-c, c), p \geq 1$, then the systems $\left\{f_{n}^{+}\right\}_{n \in N},\left\{f_{n}^{-}\right\}_{n \in N}\left(1 \cup\left\{f_{n}^{+}\right\}_{n \in N} ;\left\{f_{n}^{-}\right\}_{n \in N}\right)$ are complete in $\left.L_{p}(a, b) ; 2\right)$ 
it follows from the minimality of the systems $\left\{f_{n}^{+}\right\}_{n \in N},\left\{f_{n}^{-}\right\}_{n \in N}\left(1 \cup\left\{f_{n}^{+}\right\}_{n \in N} ;\left\{f_{n}^{-}\right\}_{n \in N}\right)$ in $L_{p}(a, b), p \geq 1$, that the systems $\left(8_{1}\right)\left(\left(8_{2}\right)\right)$ are minimal in $L_{p}(-c, c)$.

In fact, let us take $\forall f \in L_{q}(a, b)$ and suppose $F^{+}\left( \pm \omega_{k}(t)\right) \equiv f(t), \quad F^{-}\left( \pm \omega_{k}(t)\right) \equiv-f(t), \forall t \in(a, b), k=\overline{1, r}$.

We have

$$
\begin{gathered}
\int_{I_{\omega}^{+} \cup I_{\omega}^{-}} F_{n}(\tau) \overline{F^{ \pm}(\tau)} d \tau=\sum_{k=1}^{r} \int_{\omega_{k}(a)}^{\omega_{k}(b)}\left[\omega_{k}^{-1}(\tau)\right]^{\prime} b_{k}^{(1)}\left(\omega_{k}^{-1}(\tau)\right) x_{n}^{+}(\tau) \overline{F^{ \pm}(\tau)} d \tau+ \\
+\sum_{k=1}^{r} \int_{\omega_{k}(a)}^{\omega_{k}(b)}\left[\omega_{k}^{-1}(\tau)\right]^{\prime} b_{k}^{(2)}\left(\omega_{k}^{-1}(\tau)\right) x_{n}^{-}(-\tau) \overline{F^{ \pm}(-\tau)} d \tau= \\
=\int_{a}^{b} \sum_{k=1}^{r} b_{k}^{(1)}(t) x_{n}^{+}\left(\omega_{k}(t)\right) \overline{f(t)} d t \pm \int_{a}^{b} \sum_{k=1}^{r} b_{k}^{(2)}(t) x_{n}^{-}\left(-\omega_{k}(t)\right) \overline{f(t)} d t=\int_{a}^{b} f_{n}^{ \pm}(t) \overline{f(t)} d t, n \in N .
\end{gathered}
$$

Thus

Similarly we establish

$$
\int_{I_{\omega}^{+} \cup I_{\omega}^{-}} F_{n}(\tau) \overline{F^{ \pm}(\tau)} d \tau=\int_{a}^{b} f_{n}^{ \pm}(t) \overline{f(t)} d t, \quad n \in N .
$$

$$
\int_{\substack{I_{\omega}^{+} \cup I_{\omega}^{-} \\ F_{n}}}(-\tau) \overline{F^{ \pm}(\tau)} d \tau= \pm \int_{a}^{b} f_{n}^{ \pm}(t) \overline{f(t)} d t, \quad n \in N .
$$

We derive from the relations (9) and (10) that the completeness of the system $\left(8_{1}\right)\left(\left(8_{2}\right)\right)$ in $L_{p}(-c, c)$ implies the completeness of each of the systems $\left\{f_{n}^{ \pm}\right\}_{n \in N}\left(1 \cup\left\{f_{n}^{+}\right\}_{n \in N} ;\left\{f_{n}^{-}\right\}_{n \in N}\right)$ in $L_{p}(a, b)$.

Let us assume that the systems $\left\{f_{n}^{ \pm}\right\}_{n \in N}$ are minimal in $L_{p}(a, b)$ and $\left\{h_{n}^{ \pm}\right\}_{n \in N} \subset L_{q}(a, b)$ are the corresponding biorthogonal systems. Assume

and consider the system

$$
\tilde{h}_{n}^{ \pm}\left( \pm \omega_{k}(t)\right) \equiv \pm h_{n}^{ \pm}(t), t \in(a, b), k=\overline{1, r}
$$

$$
H_{n}^{ \pm}(\tau) \equiv \frac{1}{2}\left(\widetilde{h}_{n}^{+}(\tau) \pm \widetilde{h}_{n}^{-}(\tau)\right), \tau \in I_{\omega}^{+} \cup I_{\omega}^{-}
$$

We have

$$
\begin{aligned}
& \int_{I_{\omega}^{+} \cup I_{\omega}^{-}} F_{n}(\tau) \overline{H_{k}^{ \pm}(\tau)} d \tau=\frac{1}{2}\left[\int_{I_{\omega}^{+} \cup I_{\omega}^{-}} F_{n}(\tau) \overline{\widetilde{h}_{n}^{+}(\tau)} d \tau \pm \int_{I_{\omega}^{+} \cup I_{\omega}^{-}} F_{n}(\tau) \overline{\widetilde{h}_{n}^{-}(\tau)} d \tau\right]= \\
& =\frac{1}{2}\left[\int_{a}^{b} f_{n}^{+}(t) \overline{h_{k}^{+}(t)} d t \pm \int_{a}^{b} f_{n}^{-}(t) \overline{h_{k}^{-}(t)} d t\right]=\frac{1}{2}\left(\delta_{n k} \pm \delta_{n k}\right), \forall n, k \in N .
\end{aligned}
$$

Similarly we obtain

$$
\begin{aligned}
& \int_{I_{\omega}^{+} \cup I_{\omega}^{-}} F_{n}(-\tau) \overline{H_{k}^{ \pm}(\tau)} d \tau=\frac{1}{2}\left[\int_{I_{\omega}^{+} \cup I_{\omega}^{-}} F_{n}(-\tau) \overline{\widetilde{h}_{k}^{+}(\tau)} d \tau \pm \int_{I_{\omega}^{+} \cup I_{\omega}^{-}} F_{n}(-\tau) \overline{\widetilde{h}_{k}^{-}(\tau)} d \tau\right]= \\
& =\frac{1}{2}\left[\int_{a}^{b} f_{n}^{+}(t) \overline{h_{k}^{+}(t)} d t \mp \int_{a}^{b} f_{n}^{-}(t) \overline{h_{k}^{-}(t)} d t\right]=\frac{1}{2}\left(\delta_{n k} \mp \delta_{n k}\right), \forall n, k \in N .
\end{aligned}
$$

From these relations we obtain the minimality of system $\left(8_{1}\right)\left(\left(8_{2}\right)\right)$ in $L_{p}\left(I_{\omega}^{+} \cup I_{\omega}^{-}\right)$.

The following theorem is valid.

Theorem 5. Let the conditions $\gamma), \eta)$ be fulfilled. If the system $\left(8_{1}\right)\left(\left(8_{2}\right)\right)$ forms a basis for $L_{p}(-c, c), p \geq 1$, and 
$I_{\omega}^{c}[(0, c)]$ contains a non-trivial interval, then at least one of the systems $\left\{f_{n}^{ \pm}\right\}_{n \in N}\left(1 \cup\left\{f_{n}^{+}\right\}_{n \in N} ;\left\{f_{n}^{-}\right\}_{n \in N}\right)$ is non-minimal in $L_{p}(0, c)$.

This theorem is an analogue of Theorem 2 for double systems. The below theorem which is an analogue of Theorem 3 is valid as well.

Theorem 6. Let the condition $\gamma), \eta)$ be fulfilled and $r>1$. If the system ( $\left.8_{1}\right)\left(\left(8_{2}\right)\right)$ forms a basis for $L_{p}(-c, c), 1<p<+\infty$, then at least one of the systems $\left\{f_{n}^{ \pm}\right\}_{n \in N}\left(1 \cup\left\{f_{n}^{+}\right\}_{n \in N^{;}}\left\{f_{n}^{-}\right\}_{n \in N}\right)$ is non-minimal in $L_{p}(0, c)$.

In fact, let the systems $\left\{f_{n}^{ \pm}\right\}_{n \in N}$ be minimal in $L_{p}(a, b)$. If $m e s\left[(0, c) \backslash I_{\omega}\right]>0$, then we have a contradiction by virtue of Lemma 1. Therefore we will assume that $\operatorname{mes}\left[(0, c) \backslash I_{\omega}\right]=0$. It is evident that the systembiorthogonal to $\left(8_{1}\right)$ is defined by the relation (11). Denote by $\left\{H_{n}^{+} ; H_{n}^{-}\right\}_{n \in N} \subset L_{q}(-c, c)$ the systembiorthogonal to $\left(8_{1}\right)$. It is easily seen that $H_{n}^{-}(\tau) \equiv H_{n}^{+}(-\tau), \tau \in(-c, c)$. Let us assume $\gamma_{k}(\tau) \equiv \omega_{k}\left(\omega_{1}^{-1}(\tau)\right), k=\overline{1, r}$. From relations (11) we derive

$$
H_{n}^{ \pm}\left( \pm \omega_{k}(t)\right) \equiv H_{n}^{ \pm}\left( \pm \omega_{1}(t)\right), t \in(a, b) .
$$

Taking into account the latter relation, we have

$$
\int_{\omega_{k}(a)}^{\omega_{k}(b)} F_{n}(\tau) \overline{H_{m}^{+}}(\tau) d \tau=\int_{a}^{b} F_{n}\left(\omega_{k}(t)\right) \overline{H_{m}^{+}}\left(\omega_{1}(t)\right) \omega_{k}^{\prime}(t) d t=\int_{\omega_{1}(a)}^{\omega_{1}(b)} F_{n}\left(\gamma_{k}(\tau)\right) \omega_{k}^{\prime}\left(\omega_{1}^{-1}(\tau)\right) \overline{H_{m}^{+}}(\tau) d \tau .
$$

Hence

$$
\sum_{k=2}^{r} \int_{\omega_{k}(a)}^{\omega_{k}(b)} F_{n}(\tau) \overline{H_{m}^{+}}(\tau) d \tau=\int_{\omega_{1}(a)}^{\omega_{1}(b)} \sum_{k=2}^{r} F_{n}\left(\gamma_{k}(\tau)\right) \omega_{k}^{\prime}\left(\omega_{1}^{-1}(\tau)\right) \overline{H_{m}^{+}}(\tau) d \tau .
$$

In the same manner we get

$$
\sum_{k=2}^{r} \int_{-\omega_{k}(b)}^{-\omega_{k}(a)} F_{n}(\tau) \overline{H_{m}^{+}}(\tau) d \tau=\int_{-\omega_{1}(b)}^{-\omega_{1}(a)} \sum_{k=2}^{r} F_{n}\left(-\gamma_{k}(-\tau)\right) \omega_{k}^{\prime}\left(\omega_{1}^{-1}(-\tau)\right) \overline{H_{m}^{+}}(\tau) d \tau .
$$

Consequently

$$
\begin{gathered}
\sum_{k=2}^{r}\left[\int_{\omega_{k}(a)}^{\omega_{k}(b)} F_{n}(\tau) \overline{H_{m}^{+}}(\tau) d \tau\right. \\
\left.+\int_{-\omega_{k}(b)}^{-\omega_{k}(a)} F_{n}(\tau) \overline{H_{m}^{+}}(\tau) d \tau\right]=\int_{\omega_{1}(a)}^{\omega_{1}(b)} \sum_{k=2}^{r} F_{n}\left(\gamma_{k}(\tau)\right) \omega_{k}^{\prime}\left(\omega_{1}^{-1}(\tau)\right) \overline{H_{m}^{+}}(\tau) d \tau+ \\
+\int_{-\omega_{1}(b)}^{-\omega_{1}(a)} \sum_{k=2}^{r} F_{n}\left(-\gamma_{k}(-\tau)\right) \omega_{k}^{\prime}\left(\omega_{1}^{-1}(-\tau)\right) \overline{H_{m}^{+}}(\tau) d \tau .
\end{gathered}
$$

Continuing in the same way as we did when proving Theorem 3, we finish the proof of Theorem 6 . This theorem is an analogue of Theorem 3 for double systems. Using these two theorems, we come to the following

Corollary 2. Let $a, b \in \mathbb{C}$ be arbitrary non-trivial complex numbers. Then each of the systems $\left\{s_{n}^{-}\right\}_{n \in N}, 1 \cup\left\{s_{n}^{+}\right\}_{n \in N}$ is complete in $L_{p}\left(0, \frac{\pi}{2}\right)$, $p>1$. However, at least one of them is nonminimal in it with

$$
\begin{aligned}
& s_{n}^{-}=a \sin n t+b \sin n\left(t+\frac{\pi}{2}\right) \\
& s_{n}^{+}=a \cos n t+b \cos n\left(t+\frac{\pi}{2}\right) .
\end{aligned}
$$

In fact, denoting $\omega_{1}(t) \equiv t, \omega_{2}(t) \equiv t+\frac{\pi}{2} \quad$ and $x_{n}(t) \equiv e^{\text {int }}$, we can apply Theorem 6 to this system.

It should be noted that some relationship between the unitary and double power systems are considered in [12-14].

\section{Conclusions}

Summing up, we arrive at the following conclusions:

1) a method for constructing a basis in the direct product of Lebesgue spaces is suggested;

2 ) it is shown that if the system of functions forms a basis for Lebesgue space $L_{p}(I)$, then it is not minimal in $L_{p}(I)$ for $\operatorname{mes}(I \backslash J)>0, J \subset I$; 
3) let us reduce an example of the basis in $L_{p}(I)$, which is complete in $L_{p}(J): \operatorname{mes}(J \backslash I)>0, I \subset J$;

4) the unitary system of the form (1) is considered and some relations between the basis properties of the systems $\left\{f_{n}\right\}_{n \in N}$ and $\left\{x_{n}\right\}_{n \in N}$ is established;

5) it is proved that for $r>1$, from the basicity of the system $\left\{x_{n}\right\}_{n \in N}$ follows the nonminimality of the system $\left\{f_{n}\right\}_{n \in N}$;

6) ana logous results is obtained with respect to the double system of functions.

\section{ACKNOWLEDGEMENTS}

The authors are thankful to the reviewers for their valuable comments.

\section{REFERENCES}

[1] Zigmund A. Trigonometric series, Vol.1, Mir, Moscow, 1965 , 616 p. (Russian)

[2] Zigmund A. Trigonometric series, Vol.2, Mir, Moscow, 1965,538 p. (Russian)

[3] Edwards R. Fourier series in modern applications. v.1, Mir, Moscow, 1985, 264 p.

[4] Edwards R. Fourier series in modern applications. v.2, Mir,
Moscow, 1985, $400 \mathrm{p}$.

[5] Kaczmarz S., Steinhaus H. Theory of orthogonal series, Gosudarstv. Izdat. Fiz.-Mat. Lit., Moscow 1958, 508 p.

[6] Shkalikov A.A. A system of functions. Mat. Zametki, 18:6 (1975), pp. 855-860.

[7] Bilalov B.T. Completeness and minimality of some trigonometric functions. Diff. uravn., 1992, v..28, No1, pp. 170-173.

[8] Singer I. Bases in Banach spaces, v. 1, Springer, 1970, 673 p.

[9] Singer I. Bases in Banach spaces, v. 2, Springer, 1981, 880 p.

[10] Young R.M. An introduction to Nonharmonic Fourier series, Springer, 1980, $246 \mathrm{p}$.

[11] Heil Ch. A Basis Theory Primer, Springer, 2011, 536 p.

[12] Bilalov B.T. Necessary and sufficient condition for the completeness and minimality of the system of the form $\left\{A \varphi^{n} ; B \bar{\varphi}^{n}\right\}$, Dokl. RAN, 1992, v.322, No 6, pp. 1019-1021.

[13] Bilalov B.T. The basis properties of power systems in $L_{p}$. Sibirsk. Mat. Zh., 2006, v.47. No 1, pp. 25-36.

[14] Bilalov B.T. A system of exponential functions with shift and the Kostyuchenko problem. Sibirsk. Mat. Zh., 2009, v.50, No 2, pp. 279-288. 